\title{
Thermal conductance of the junction between single-walled carbon nanotubes
}

\author{
Lin Hu and Alan J. H. McGaughey ${ }^{a}$ \\ Department of Mechanical Engineering, Carnegie Mellon University, Pittsburgh, Pennsylvania 15213, USA
}

(Received 9 September 2014; accepted 6 November 2014; published online 12 November 2014)

\begin{abstract}
The thermal conductances of the carbon nanotube (CNT) junctions that would be found in a CNT aerogel are predicted using molecular dynamics simulations. At a temperature of $300 \mathrm{~K}$, the thermal conductance of a perpendicular junction converges to $40 \mathrm{pW} / \mathrm{K}$ as the CNT lengths approach $100 \mathrm{~nm}$. The key geometric parameter affecting the thermal conductance is the angle formed by the two CNTs. At pressures above 1 bar, the presence of a surrounding gas leads to an effective increase in the junction thermal conductance by providing a parallel path for energy flow. C 2014 AIP Publishing LLC. [http://dx.doi.org/10.1063/1.4902074]
\end{abstract}

Networks of carbon nanotubes (CNTs) (e.g., aligned films, mats, and aerogels) are candidates for use in electronic and optoelectronic devices, as thermal interface materials, and for thermal insulation. ${ }^{1}$ Thermal management is a key issue in all of these applications. Our focus here is related to single-walled CNT aerogels, which are a high-porosity material (densities $\rho$ of $6-20 \mathrm{~kg} / \mathrm{m}^{3}$ ) where the individual CNTs form a random network held together by van der Waals interactions. ${ }^{2,3}$ For a CNT aerogel in vacuum at a temperature of $300 \mathrm{~K}$, Schiffres et al. report a thermal conductivity of $0.025 \mathrm{~W} / \mathrm{m} \cdot \mathrm{K}\left(\rho=8 \mathrm{~kg} / \mathrm{m}^{3}\right.$, which corresponds to a volume fraction of 0.006), ${ }^{4}$ while Zhang et al. report a value of $0.055 \mathrm{~W} / \mathrm{m} \cdot \mathrm{K}\left(\rho=6.6 \mathrm{~kg} / \mathrm{m}^{3}\right){ }^{5}$ These values are much lower than the $12 \mathrm{~W} / \mathrm{m} \cdot \mathrm{K}$ predicted from an effective medium approximation for a random network of cylinders using a single-walled CNT thermal conductivity of $3000 \mathrm{~W} / \mathrm{m} \cdot \mathrm{K}{ }^{4}$ This result indicates that the thermal conductance of the junctions between CNTs must be taken into account when predicting thermal conductivity.

Yang et al. measured a thermal conductance of $97 \mathrm{nW} / \mathrm{K}$ at the perpendicular junction between two multiwalled CNTs with diameters of 72 and $121 \mathrm{~nm}$ at a temperature of $300 \mathrm{~K} .{ }^{6}$ Scaling this value to estimate the thermal conductance between two $(6,6)$ single-walled CNTs, as would be found in a CNT aerogel, gives $7 \mathrm{pW} / \mathrm{K}$. Recent measurements on perpendicular junctions between multiwalled CNTs with diameters of 42 to $68 \mathrm{~nm}$, however, indicate that such a scaling is not appropriate as the per unit area conductance shows a diameter dependence. ${ }^{7}$ Some estimates of the thermal conductance of the junction between single walled CNTs from thermal conductivity measurements on different network materials suggest values between 2 and $12 \mathrm{pW} / \mathrm{K},{ }^{4,8,9}$ while Zhang et al. estimate $60 \mathrm{pW} / \mathrm{K} .^{5}$

Molecular dynamics (MD) simulations ${ }^{8,10}$ and atomistic Green's function calculations ${ }^{8,11}$ of perpendicular junctions between single-walled CNTs predict thermal conductances between 10 and $100 \mathrm{pW} / \mathrm{K}$ at a temperature of $300 \mathrm{~K}$ that increase with increasing CNT diameter. The origin of these larger values compared to the majority of

${ }^{\text {a)} E l e c t r o n i c ~ m a i l: ~ m c g a u g h e y @ c m u . e d u ~}$ the experimental estimates will be discussed later in the paper. We note that a junction thermal conductance of $100 \mathrm{pW} / \mathrm{K}$ is equivalent to that through a $2.5 \mu \mathrm{m}$ long singlewalled CNT with thermal conductivity of $3000 \mathrm{~W} / \mathrm{m} \cdot \mathrm{K}$. Given that the average length of the CNTs in a CNT aerogel is $\sim 1 \mu \mathrm{m}$ (Ref. 4) and that the average distance between junctions ranges from 20 to $90 \mathrm{~nm}$ (depending on the density), ${ }^{12}$ it is clear that the junctions play an important role in the thermal transport.

The objective of this study is to quantify geometrical effects on the thermal conductance of junctions that would be found in a single-walled CNT aerogel. The effects of the CNT lengths, in-plane angle, initial separation distance, and axial orientations are investigated. We also consider how thermal transport is affected by closely spaced junctions and a surrounding gas. These thermal conductances will be useful in parameterizing network-level thermal transport models for predicting thermal conductivity, which are too large for a full atomistic treatment. ${ }^{13,14}$

We use MD simulations to predict the thermal conductance of the junction between two $(6,6)$ single-walled CNTs (diameter $D$ of $0.81 \mathrm{~nm}$ ) using the setup shown in Fig. 1(a). For a given configuration, each CNT has the same length $L$ and the junction is placed at the middle of each CNT. For computational convenience, the 24 atoms at each end of both CNTs are fixed. ${ }^{15}$ The geometry of the junction can be described by the in-plane angle between the two CNTs $\theta$, the distance between them $d$, and the axial rotational orientation $\phi$. The atomic interactions within each CNT and between CNTs are described by the adaptive intermolecular reactive empirical bond-order (AIREBO) potential. ${ }^{16}$ The MD simulations are performed using LAMMPS $^{17}$ with a time step of $1 \mathrm{fs}$. For the benchmarking simulations, unless noted, $L=20 \mathrm{~nm}, \theta=90^{\circ}, d=3.4 \AA$ (the equilibrium separation), and $\phi=0^{\circ}$.

For each configuration studied, the system is first equilibrated in the canonical ensemble at a temperature of $300 \mathrm{~K}$ for 200 ps using the Nose-Hoover thermostat. The thermostat is then turned off and a constant heat source of magnitude $q$ is applied at one end of one CNT (the hot reservoir) using velocity rescaling. A heat sink of the same magnitude is applied at one end of the other CNT (the cold reservoir). 
(a)

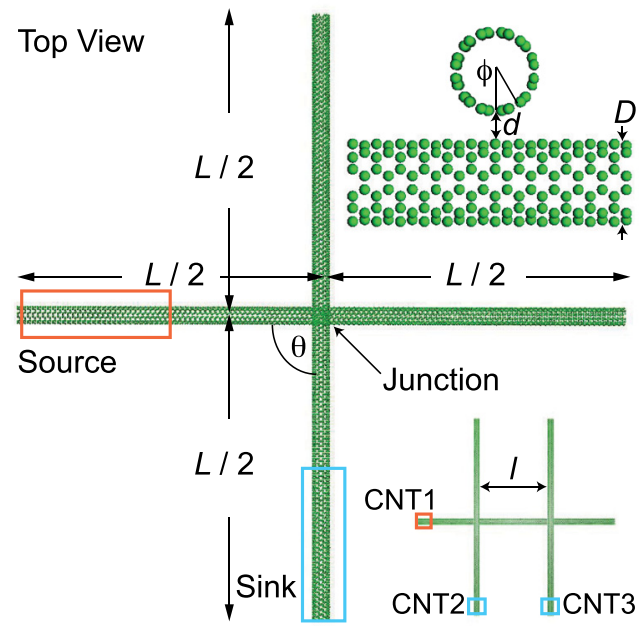

Side View

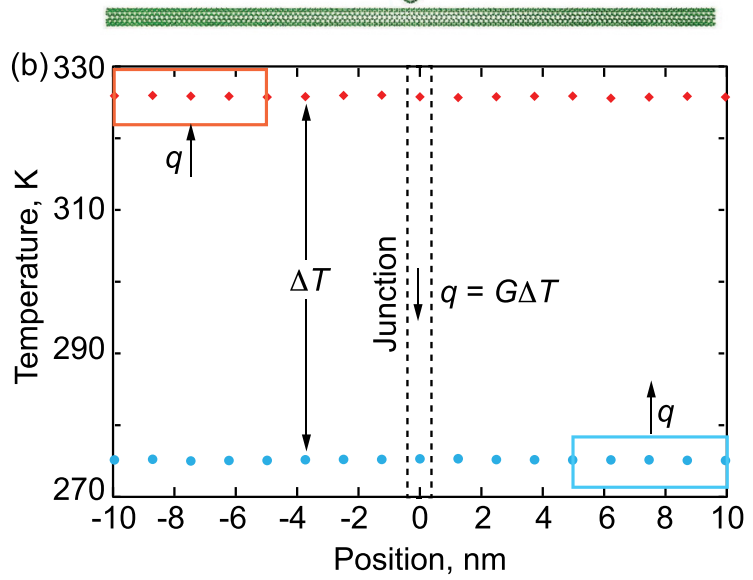

FIG. 1. (a) Molecular dynamics simulation setup used to predict the junction thermal conductance. (b) Typical steady-state temperature profiles in the two CNTs for $L=20 \mathrm{~nm}$.

Each reservoir is $5 \mathrm{~nm}$ long and contains 480 atoms. Steady state is reached after $5 \mathrm{~ns}$ and the temperatures in 20 equally sized bins along each CNT are calculated and averaged over 10 to $20 \mathrm{~ns}$, depending on the system size. A typical temperature profile is shown in Fig. 1(b). The temperature along each CNT is uniform and there is only a temperature drop, $\Delta T$, at the junction, confirming that the thermal resistance within each CNT is negligible. The applied heat fluxes are in the range of 1 to $100 \mathrm{nW}$ depending on the system configuration, leading to $\Delta T$ values between 15 and $60 \mathrm{~K}$. The junction thermal conductance $G$ is then obtained from $G=q / \Delta T$. By performing the calculations on subsets of the total data collection period, we estimate the uncertainty in the thermal conductance predictions to be $5 \%$.

To determine the effect of the axial rotational orientations of the individual CNTs, the junction thermal conductance was predicted for $0^{\circ} \leq \phi \leq 30^{\circ}$ for both CNTs, which covers the full potential energy landscape. The thermal conductances show no trend with $\phi$ and vary within $\pm 7 \%$ of the mean value, which is on the order of the estimated prediction uncertainty.

The distance between the two CNTs is important due to the fast decay of van der Waals interactions. To investigate this effect, we considered two CNTs of length $90 \mathrm{~nm}$. With the ends of both CNTs fixed, the initial separation distance $d$ was varied from $1.8 \AA$ to $5.4 \AA$ and the system was equilibrated. In all cases, the final separation distance is within $1.5 \%$ of the equilibrium separation distance of $3.4 \AA$ and the thermal conductance is within $5 \%$ of that for $d=3.4 \AA$. As such, for all subsequent simulations, the initial separation distance is set to $3.4 \AA$.

In a CNT network structure, multiple junctions will exist on one CNT. To test if multiple junctions on the same CNT affect the individual thermal conductances, we built a simulation cell with the structure shown in the bottom right inset to Fig. 1(a), where CNT1 forms junctions with CNT2 and CNT3 that are separated by a distance $l$. The CNTs all have the same length and $L$ values of 30,60 , and $90 \mathrm{~nm}$ are considered. The heat source is placed at the left end of CNT1 and a heat sink (removing half of the energy supplied by the source) is placed at one end of each of CNT2 and CNT3. The thermal conductances of the two junctions are provided in Table I along with the results for a single junction. The closely spaced junctions show little variation with compared to the single junction, with a decrease of about $20 \%$ for $l=10$ and $20 \mathrm{~nm}$ for the $L=60 \mathrm{~nm}$ system. This result indicates that adjacent junctions are not significantly affected by each other at these separation distances, which are typical of what will be found in a CNT aerogel. ${ }^{12}$ Evans et al. reached a similar conclusion for two perpendicular $(10,10)$ CNTs in a system with periodic boundary conditions. ${ }^{10}$ For a junction built from two parallel $(10,10)$ CNTs separated by $0.8 \mathrm{~nm}$ and connected by a third, short $(10,10)$ CNT, Prasher et al. ${ }^{8}$ found that the thermal conductance between the two parallel CNTs was reduced by an order of magnitude compared to a single perpendicular junction. Such closely spaced junctions are unlikely to be found in low-density CNT networks, but may be present in the packed beds studied by Prasher et al., which have densities on the order of $100 \mathrm{~kg} / \mathrm{m}^{3}$.

The CNT length for the single junction system was then varied from 20 to $200 \mathrm{~nm}$ and the resulting thermal conductances are plotted in Fig. 2. The junction thermal conductance increases with increasing CNT length, converging to $40 \mathrm{pW} / \mathrm{K}$ as the length approaches $100 \mathrm{~nm}$, which is an order of magnitude shorter than the average CNT length in the aerogel. This thermal conductance value is about half of that reported by Chalopin et al. for two infinite perpendicular $(6,6)$ CNTs using atomistic Green's functions calculations. ${ }^{11}$ We note that they used a stronger non-bonded Lennard-Jones carbon-carbon interaction (length scale $0.228 \mathrm{~nm}$ and energy scale $4.41 \mathrm{meV}$ ) as compared to that in the AIREBO potential (length scale $0.340 \mathrm{~nm}$ and energy scale $2.84 \mathrm{meV}$ ). Upon repeating our $L=90 \mathrm{~nm}$ simulation using the Lennard-Jones parameters of Chalopin et al., we predicted a junction thermal conductance of $80 \mathrm{pW} / \mathrm{K}$, consistent with their result.

Albeit using different potentials, Chalopin et al. found that the transmission function between the two perpendicular

TABLE I. Thermal conductance of a single junction and two closely-spaced junctions (in pW/K), as per the geometries shown in Fig. 1(a).

\begin{tabular}{lcccc}
\hline \hline$L(\mathrm{~nm})$ & Single junction & $l=10 \mathrm{~nm}$ & $20 \mathrm{~nm}$ & $30 \mathrm{~nm}$ \\
\hline 30 & 31 & 29,30 & - & - \\
60 & 38 & 32,34 & 33,34 & 35,38 \\
90 & 38 & 36,38 & 37,37 & 38,41 \\
\hline \hline
\end{tabular}




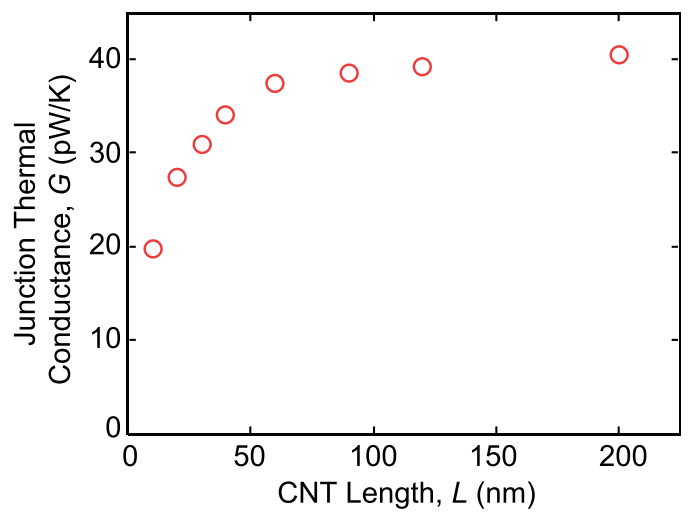

FIG. 2. Junction thermal conductance plotted as a function of CNT length.

$(6,6)$ CNTs was only non-zero below phonon frequencies of $25 \mathrm{THz}$, corresponding to acoustic phonons and low-lying optical phonons. As the CNTs in our simulations get longer, more low-frequency modes emerge until convergence in the junction thermal conductance is reached. Evans et al. ${ }^{10}$ found that the thermal conductance of their perpendicular $(10,10)$ CNTs with periodic boundary conditions converged for CNT lengths of $10 \mathrm{~nm}$. They explain the convergence using a similar argument as that of Chalopin et al., noting that high-frequency phonons must couple to low-frequency phonons to transfer their energy across the junction. The much shorter convergence length predicted by Evans et al. compared to our prediction may be a result of their use of periodic boundary conditions.

To this point, all our simulations used perpendicular CNTs. In a random dispersion of CNTs, however, the angle $\theta$ will take on a range of values. Previous results on different CNT configurations ${ }^{18}$ and diameters ${ }^{10}$ found that the junction thermal conductance increases as the contact area between the two CNTs increases, which is confirmed by our predictions plotted in Fig. 3 for two different CNT lengths. These results also indicate that the length independence above $90 \mathrm{~nm}$ shown in Fig. 2 for $\theta=90^{\circ}$ holds for different angles.

For a network of randomly oriented CNTs, Chalopin et al. derived the following expression for thermal conductivity: ${ }^{11}$

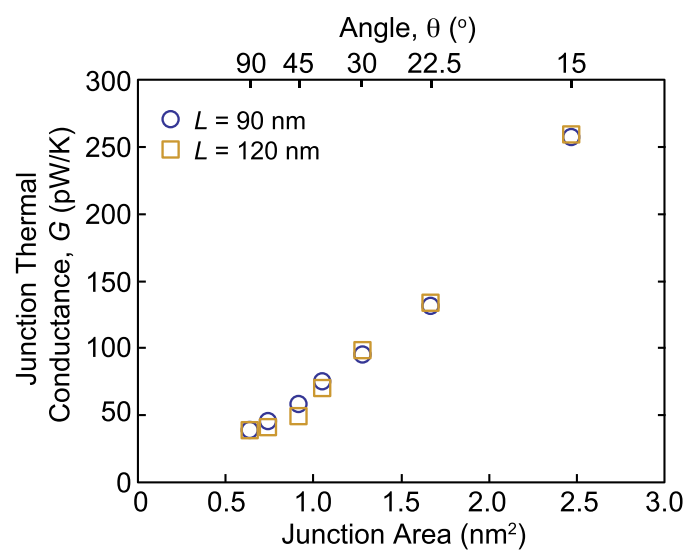

FIG. 3. Junction thermal conductance plotted as a function of the junction area $\left(D^{2} / \sin \theta\right)$ and angle.

$$
k=\frac{5 G L \rho}{54 \pi D \rho_{g}},
$$

where $\rho_{g}$ is the planar density of graphene, $7.6 \times 10^{-7} \mathrm{~kg} / \mathrm{m}^{2}$. For a CNT aerogel, taking $G=40 \mathrm{pW} / \mathrm{K}$ (Fig. 2), $L=1 \mu \mathrm{m}$, $\rho=8 \mathrm{~kg} / \mathrm{m}^{3}$, and $D=0.81 \mathrm{~nm}$, we obtain $0.015 \mathrm{~W} / \mathrm{m} \cdot \mathrm{K}$, which is of the same order of magnitude as the measurements of Schiffres et al. ${ }^{4}$ and Zhang et al. ${ }^{5}$ The prediction is likely an underestimate, as the average junction thermal conductance will be higher due to different CNT orientations, as shown in Fig. 3.

Consistent with previous atomistic modeling efforts, we find junction thermal conductances higher than the majority of estimates extracted from experimental measurements. At the same time, our prediction of thermal conductivity from Eq. (1) gives a slight under prediction of measurements on a CNT aerogel. Possible reasons for these seemingly contradictory findings include: (1) Some experimental junctions may contain traces of molecules used during synthesis that would inhibit the thermal coupling between the CNTs. (2) The models used to extract estimates from the experiments may not be representative of the structure of the CNT network. We note that the high junction thermal conductance estimate from the experiments of Zhang et al. (60 pW/K) was based on Eq. (1). (3) Molecular dynamics simulations, which are classical, may over predict the contribution of high-frequency modes. We note, however, that the atomistic Green's function calculations by Chalopin et al. used quantum statistics and still predicted much higher thermal conductances than the majority of the experimental estimates. (iv) The potentials chosen to model the atomic interactions. Given that the CNTs are found to be isothermal [see Fig. 1(b)], it is the non-bonded interaction that is important. Further effort may be required to better specify this interaction.

A CNT aerogel may be placed in a gas environment for a particular application. To investigate how gas molecules affect the junction thermal conductance, we considered a perpendicular junction formed by two $20 \mathrm{~nm}$ long CNTs in the presence of helium or argon between pressures of 0 and $12.5 \mathrm{bar}$. The MD simulation box was of size $20 \times 20 \times 20 \mathrm{~nm}^{3}$ and had periodic boundary conditions in all directions. The carbon atoms at the system boundaries are fixed. The gas-carbon and gas-gas interactions are modeled using Lennard-Jones potentials. ${ }^{15}$ The resulting junction thermal conductances are plotted in Fig. 4. There is no difference between the results for helium and argon within the prediction uncertainty, which we attribute to helium having a smaller energy accommodation coefficient than argon but a higher collision rate. ${ }^{19}$

The thermal conductance increases with pressure as more gas atoms carry thermal energy indirectly from the hot CNT to the cold CNT through a parallel path to the direct CNT-CNT interaction. For pressures above 8 bar, the junction thermal conductance becomes independent of pressure. We attribute this result to an increase of collisions between gas atoms (i.e., a reduction of their mean free path) such that the number that travels directly from one CNT to the other no longer increases with pressure. For gas filling pressures less than 1 bar, Schiffres et al. attribute the increase of thermal conductivity of a CNT aerogel with increasing pressure 


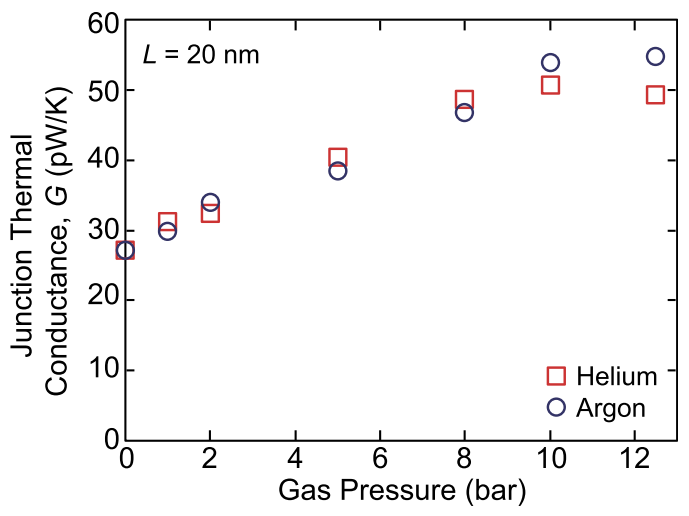

FIG. 4. Junction thermal conductance in the presence of helium and argon plotted as a function of gas pressure.

to the thermal energy carried by the gas molecules themselves and not to an increase in the junction thermal conductance. ${ }^{4}$ Consistent with this interpretation, we do not predict any noticeable enhancement of the junction thermal conductance at pressures below 1 bar.

In summary, we used MD simulations to study geometrical effects on the thermal conductance at the junction between two $(6,6)$ CNTs. Our results indicate that for low-density CNT networks (e.g., a CNT aerogel), the angle between the CNTs has the most pronounced effect on the thermal conductance, while the lengths, axial rotational angles, and the presence of other junctions on the same CNT have a small effect. Using a simple model [Eq. (1)], ${ }^{11}$ we predict a thermal conductivity for a CNT aerogel of the same order of magnitude as previous experimental measurements. ${ }^{4,5}$ These thermal conductances will be useful as input to network-level thermal transport models.

This work was supported by NSF Award CBET0933510.

${ }^{1}$ A. M. Marconnet, M. A. Panzer, and K. E. Goodson, Rev. Mod. Phys. 85, 1295 (2013).

${ }^{2}$ M. B. Bryning, D. E. Milkie, M. F. Islam, L. A. Hough, J. M. Kikkawa, and A. G. Yodh, Adv. Mater. 19, 661 (2007).

${ }^{3}$ K. H. Kim, M. Vural, and M. F. Islam, Adv. Mater. 23, 2865 (2011).

${ }^{4}$ S. N. Schiffres, K. H. Kim, L. Hu, A. J. H. McGaughey, M. F. Islam, and J. A. Malen, Adv. Funct. Mater. 22, 5251 (2012).

${ }^{5}$ K. J. Zhang, A. Yadav, K. H. Kim, Y. Oh, M. F. Islam, C. Uher, and K. P. Pipe, Adv. Mater. 25, 2926 (2013).

${ }^{6}$ J. Yang, S. Waltermire, Y. Chen, A. A. Zinn, T. T. Xu, and D. Li, Appl. Phys. Lett. 96, 023109 (2010).

${ }^{7}$ J. Yang, M. Shen, Y. Yang, W. J. Evans, Z. Wei, W. Chen, A. A. Zinn, Y. Chen, R. Prasher, T. T. Xu, P. Keblinski, and D. Li, Phys. Rev. Lett. 112, 205901 (2014).

${ }^{8}$ R. S. Prasher, X. J. Hu, Y. Chalopin, N. Mingo, K. Lofgreen, S. Volz, F. Cleri, and P. Keblinski, Phys. Rev. Lett. 102, 105901 (2009).

${ }^{9}$ D. Estrada and E. Pop, Appl. Phys. Lett. 98, 073102 (2011).

${ }^{10}$ W. J. Evans, M. Shen, and P. Keblinski, Appl. Phys. Lett. 100, 261908 (2012).

${ }^{11}$ Y. Chalopin, S. Volz, and N. Mingo, J. Appl. Phys. 105, 084301 (2009).

${ }^{12}$ L. A. Hough, M. F. Islam, B. Hammouda, A. G. Yodh, and P. A. Heiney, Nano Lett. 6, 313 (2006).

${ }^{13}$ A. N. Volkov and L. V. Zhigilei, Phys. Rev. Lett. 104, 215902 (2010).

${ }^{14}$ A. N. Volkov, R. N. Salaway, and L. V. Zhigilei, J. Appl. Phys. 114, 104301 (2013).

${ }^{15}$ See supplementary material at http://dx.doi.org/10.1063/1.4902074 for a discussion of the boundary conditions and the values of the gas-carbon and gas-gas Lennard-Jones parameters.

${ }^{16}$ S. J. Stuart, A. B. Tutein, and J. A. Harrison, J. Chem. Phys. 112, 6472 (2000).

${ }^{17}$ S. Plimpton, J. Comput. Phys. 117, 1 (1995).

${ }^{18}$ H. Zhong and J. R. Lukes, Phys. Rev. B 74, 125403 (2006).

${ }^{19}$ L. Hu and A. J. H. McGaughey, J. Phys. Chem. C 117, 18804 (2013). 